\title{
Voltage control of ferromagnetic resonance
}

\author{
Ziyao Zhou*, Bin Peng*, Mingmin Zhu* and \\ Ming Liu* ${ }^{*} \dagger$ \\ *Electronic Materials Research Laboratory \\ Key Laboratory of the Ministry of Education \& \\ International Center for Dielectric Research, \\ Xi'an Jiaotong University \\ Xi'an 710049, P. R. China \\ †Mingliu@mail.xjtu.edu.cn
}

Received 8 December 2015; Revised 4 April 2016; Accepted 6 April 2016; Published 16 May 2016

\begin{abstract}
Voltage control of magnetism in multiferroics, where the ferromagnetism and ferroelectricity are simultaneously exhibiting, is of great importance to achieve compact, fast and energy efficient voltage controllable magnetic/microwave devices. Particularly, these devices are widely used in radar, aircraft, cell phones and satellites, where volume, response time and energy consumption is critical. Researchers realized electric field tuning of magnetic properties like magnetization, magnetic anisotropy and permeability in varied multiferroic heterostructures such as bulk, thin films and nanostructure by different magnetoelectric (ME) coupling mechanism: strain/stress, interfacial charge, spin-electromagnetic (EM) coupling and exchange coupling, etc. In this review, we focus on voltage control of ferromagnetic resonance (FMR) in multiferroics. ME coupling-induced FMR change is critical in microwave devices, where the electric field tuning of magnetic effective anisotropic field determines the tunability of the performance of microwave devices. Experimentally, FMR measurement technique is also an important method to determine the small effective magnetic field change in small amount of magnetic material precisely due to its high sensitivity and to reveal the deep science of multiferroics, especially, voltage control of magnetism in novel mechanisms like interfacial charge, spin-EM coupling and exchange coupling.
\end{abstract}

Keywords: Multiferroics; magnetoelectric coupling; ferromagnetic resonance; voltage control; thin films.

\section{Introduction}

Multiferroic materials ${ }^{1-9}$ are the materials that combine two or more of the ferroic properties including ferroelectricity, ferromagnetism and ferroelasticity, etc. Both single-phase multiferroic materials and multiferroic composites attract lots of researchers' efforts to obtain magnetic field (H-field) control of electric polarization (direct magnetoelectric (ME) effect), or electric field (E-Field) manipulation of magnetization (converse ME effect) through ME coupling and then lead to varied multiferroic devices. Multiferroic composites, for instance, layered ferromagnetic/ferroelectric and ferromagnetic/piezoelectric multiferroic heterostructures, compared to single-phase multiferroics, has much stronger ME coupling strength that is $1-2$ order higher than singlephase multiferroics.

The ever increasing demand of smaller, faster, low energy consumption tunable electronics ${ }^{10,11}$ such as antennas, ${ }^{12-14}$ inductors, ${ }^{15-17}$ voltage tunable RF/microwave signal processing devices, ${ }^{18-21} \mathrm{ME}$ sensors, ${ }^{22,23}$ energy harvesters, ${ }^{24}$ filters, ${ }^{25,26}$ etc., has propelled the discovery of controlling magnetic properties such as magnetization, magnetic anisotropy and permeability through energy conservation between electrical energy and magnetic energy. Controlling magnetic properties through electric field without magnetic field from bulky, noisy and energy consumption electromagnetics (EM) is critical in these devices. For example, data storage devices are recently becoming so small that the local magnetic field that is writing a single bit is easily influencing the neighboring bits, introducing instabilities in the stored data. To overcome the issue is to create new material composites and functionalities while integrating them into nonvolatile, lightweight and energy-efficient memory devices. In addition, the E-field controllable memory devices are much faster due to quicker response of E-field tuning magnetism in multiferroics.

Within state-of-the-art RF/microwave devices, materialsdependent ferromagnetic resonance (FMR) frequencies of magnetic materials can be manipulated by electromagnets; therefore, limiting their deployment in radar, satellite, aircraft, cell phones and portable communication devices in which

This is an Open Access article published by World Scientific Publishing Company. It is distributed under the terms of the Creative Commons Attribution 4.0 (CC-BY) License. Further distribution of this work is permitted, provided the original work is properly cited. 
mass, volume and power consumption are considered at a premium. Thus, tuning the FMR of magnetic materials by electric field is of great importance to achieve next generation voltage controllable $\mathrm{RF} /$ microwave devices. In this review paper, we will discuss the recent progresses of voltage control of FMR in different multiferroic composites and by different ME coupling mechanisms.

Conventional ME coupling in multiferroic heterostructures were realized through strain/stress interaction between ferromagnetic phase and ferroelectric phase. ${ }^{27-49}$ Lots of layered strain/stress dominated multiferroic heterostructures such as bulk/bulk, ${ }^{27-31}$ thin film/bulk ${ }^{32-45}$ and thin film/thin film ${ }^{47}$ magnetic/ferroelectric or magnetic/piezoelectric heterostructures were demonstrated in this paper. By applying voltage across the ferroelectric/piezoelectric layer, the ferroelectric/piezoelectric layer generates a large strain/stress energy change that influence the magnetic layer, therefore, change the effective magnetic anisotropy energy which lead to an effective FMR field or frequency change. The pioneering work began with YIG/PZT, ${ }^{27}$ Terfenol-D/ PMN-PT ${ }^{30}$ layered bonding layered magnetic/ferroelectric multiferroic devices. Nevertheless, the FMR tunability $(<200 \mathrm{MHz})^{25-27}$ is limited by bulky magnetic slab and bonding interface. Further, researchers deposited nanoscale magnetic thin film onto well-polished ferroelectric/piezoelectric slab such as Ni/PMN-PT, ${ }^{32}$ FeGaB/PZT, ${ }^{36}$ FeGaB/ PZN-PT, ${ }^{42}$ Terfenol-D/PZN-PT, ${ }^{43}$ etc. The well-established interface and magnetic thin film allows the strain/stress energy transferred and affected magnetic energy efficiently. As a result, giant FMR tunability $(>3500 \mathrm{Oe},>13 \mathrm{GHz})^{42,43}$ was achieved in these heterostructures. For real Si-based integrated circuit, piezoelectric thin films $(\mathrm{ZnO})^{46,47}$ with lower fabrication temperature $\left(<90^{\circ} \mathrm{C}\right)$ are considered and thin film multiferroic heterostructure of $\mathrm{Fe}_{3} \mathrm{O}_{4} / \mathrm{ZnO}^{47}$ was studied. The tunability was also limited by sample clamping effect.

To conquer the limitation of thin film multiferroic heterostructure, other mechanisms like EM-spin wave coupling, interfacial charge and exchange coupling were developed and studied. ${ }^{49,50}$ In YIG/BSTO thin film heterostructure, 5-6 Oe small FMR field change was observed by EM-spin wave coupling. ${ }^{49}$ Interfacial charge-induced ME coupling in ultrathin magnetic film $(\sim 1 \mathrm{~nm})$ also do not suffer from sample clamping effect, ${ }^{51-59}$ for example, $\sim 60 \mathrm{Oe}\left(\mathrm{NiFe} / \mathrm{SrTiO}_{3}\right)^{58}$ and $\sim 200$ Oe $(\mathrm{NiFe} / \mathrm{PMN}-\mathrm{PT})^{59}$ FMR field tunability was demonstrated. FMR tunability was also shown in $\mathrm{MgO}$ ultrathin film magnetic tunneling junctions (MTJ). ${ }^{60,61}$ Recently, a reversible, nonvolatile FMR field switch of 60 Oe from $\mathrm{CoFe} / \mathrm{BiFeO}_{3}$ (BFO) system ${ }^{62-73}$ was shown and all these achievements pave a way to voltage controllable $\mathrm{RF} /$ microwave military/civilian devices. Beyond voltage control of FMR, in contrast, the FMR measurement technique, for example, electron spin resonance (ESR) ${ }^{73}$ method is a unique way to determine very small the E-field control of magnetic anisotropic field change in small amount of magnetic material in multiferroic heterostructures.

\section{Voltage Control of FMR through Strain/Stress- Induced ME Coupling in Different Multiferroic Heterostructures}

In many researches, controlling of strain/stress of piezoelectric/ferroelectric substrates is the ideal way to change magnetic properties of magnetic components that are coupled to piezoelectric/ferroelectric substrates. ${ }^{32-48}$ The E-field was applied across substrate and the magnetic field was applied along the in-plane (IP) direction, vertical to the microwave propagation. As an E-field is applied, the piezoelectric substrate undergoes a tensile or compressive deformation and that strain can be coherently transferred to magnetic films resulting in an effective magnetic field $H_{\text {eff }}$ through the magnetoelastic effect. $H_{\text {eff }}$ can be written as:

$$
H_{\mathrm{eff}}=3 \lambda_{S} Y d E / \mu_{0} M_{S}
$$

where $\lambda s$ is the magnetostriction constant of magnetic materials, $Y$ is Young's modulus, $d$ is the piezoelectric coefficient of ferroelectric substrate and $M_{S}$ is the magnetization. ${ }^{33,34}$ To obtain strong ME coupling, large $\lambda_{S}, d$ and small $M_{S}$ are needed. By using Kittel equation, FMR frequency can be calculated as ${ }^{33,34}$ :

$$
f=\gamma \sqrt{\left(H_{r}+H_{\text {eff }}\right)\left(H_{r}+H_{\text {eff }}+4 \pi M_{S}\right)},
$$

where $H_{r}$ is the resonance field, $f$ is microwave frequency and $\gamma=2.8 \mathrm{MHz} / \mathrm{Oe}$ is gyromagnetic ratio. By controlling the effective field $H_{\text {eff }}$, the FMR frequency, as a consequence, can be easily manipulated.

\subsection{FMR tuning in bulk multiferroic heterostructures}

High permeability and high permittivity multiferroic composites with strong $\mathrm{ME}$ coupling at $\mathrm{RF} / \mathrm{microwave}$ frequencies provide great opportunities for future compact, lightweight and power efficient voltage tunable RF/ microwave devices. Numerous multiferroic devices such as antennas, ${ }^{12-14}$ inductors, ${ }^{15-17}$ voltage tunable RF/microwave signal processing devices, ${ }^{18-21} \mathrm{ME}$ sensors, ${ }^{22,23}$ energy harvesters, ${ }^{24}$ filters, ${ }^{25,26}$ etc., are developed. For example, the microstrip bandpass filter is based on FMR of ferrite component. The device operating at $5-10 \mathrm{GHz}$ can be tuned over a wide frequency band by a bias magnetic field and over a narrow band with a voltage applied across piezoelectric component. The voltage tuning of the device is possible through ME interactions that are mediated by mechanical deformation and manifests as a shift in FMR. Data on tuning range, insertion loss and device characteristics are presented for filters with single- and double-ME resonators.

Figure 1 shows a dual $\mathrm{H}$-field and E-field controllable Niferrite/PMN-PT multiferroic bandpass filter. ${ }^{19} \mathrm{H}$-field bias of 0,75 and 100 Oe were applied along the $y$-axis of the bandpass filter and E-field from 1 to $9 \mathrm{kV} / \mathrm{cm}$ is applied along the thickness direction of the PMT-PT slab in bandpass filter. As shown in Fig. 1(a), a maximum ME tunability of 270 


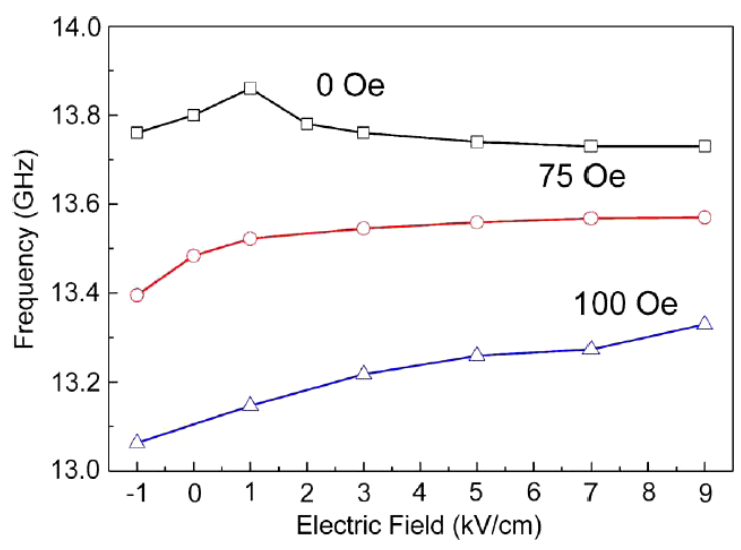

(a)

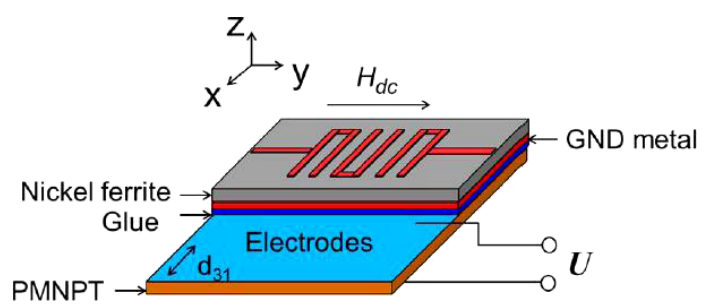

(b)

Fig. 1. (a) Measured E-field tunable operating frequency range of the bandpass filter under different magnetic bias fields, showing the dual E- and H-field tunability. (b) Diagram showing the device with ferrite/PMN-PT multiferroic heterostructure. ${ }^{19}$

$\mathrm{MHz}(2.1 \%)$ was obtained at $100 \mathrm{Oe} \mathrm{H}$-field bias field, when E-field is applied from $3 \mathrm{kV} / \mathrm{cm}$ to $9 \mathrm{kV} / \mathrm{cm}$. The central frequency was shifted from $13.22 \mathrm{GHz}$ to $13.33 \mathrm{GHz}$ correspondingly.

To further increase the tunability of bulk ME laminates, according to Eq. (1), piezoelectric substrates with larger piezoelectric coefficient $d$ should be considered. Tatarenko and Bichurin designed and fabricated layered YIG/PMN-PT structures, ${ }^{29}$ see Fig. 2, in which the PMN-PT has stronger piezoelectric property. The ME coupling theory and experiment of attenuators, bandpass filters and phase shifters were discussed. Figure 2 shows the design of YIG/PMN-PT attenuator and the tunability of insertion loss from $26 \mathrm{~dB}$ to $2 \mathrm{~dB}$ at $7.251 \mathrm{GHz}$ by applying E-field across the PMN-PT substrate. Other multiferroic devices like bandpass filter and phase shifter were also discussed: tunability of $25 \mathrm{MHz}$ in bandpass filter at $7.36 \mathrm{GHz}$ frequency achieved, meanwhile, a $30-40^{\circ}$ phase shift around the frequency range of $6-9 \mathrm{GHz}$ was obtained in YIG/PMN-PT multiferroic composites.

\subsection{FMR tuning in thin film/bulk multiferroic heterostructures}

Eventually, bulk multiferroic composites suffer from limited tunability due to sample clamping effect of magnetic bulk component as well as the poor adhesion condition of interface

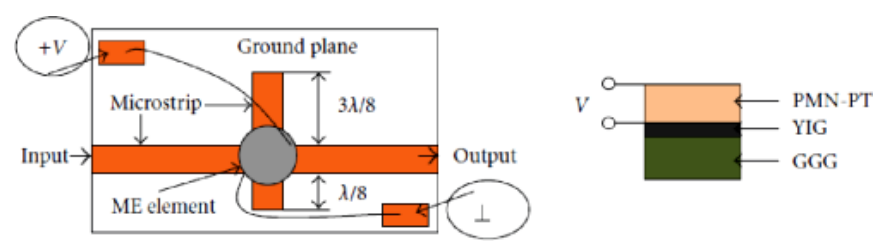

(a)

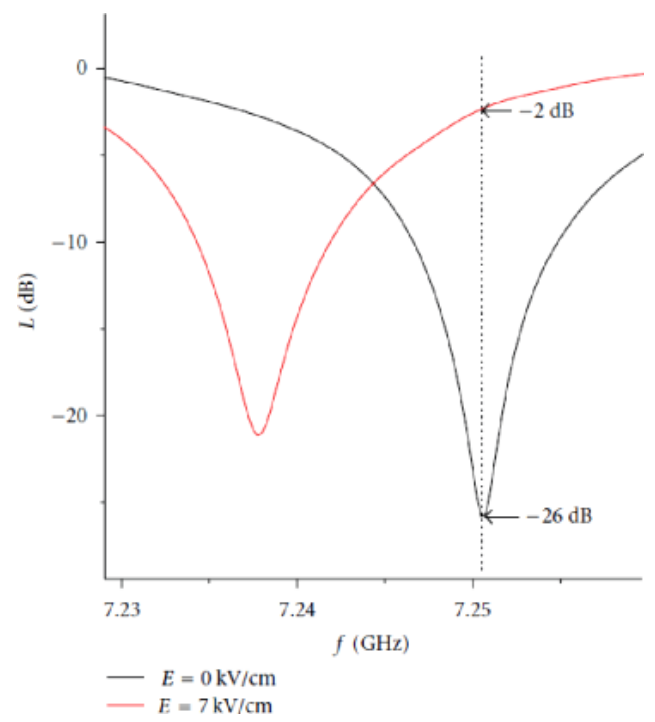

(b)

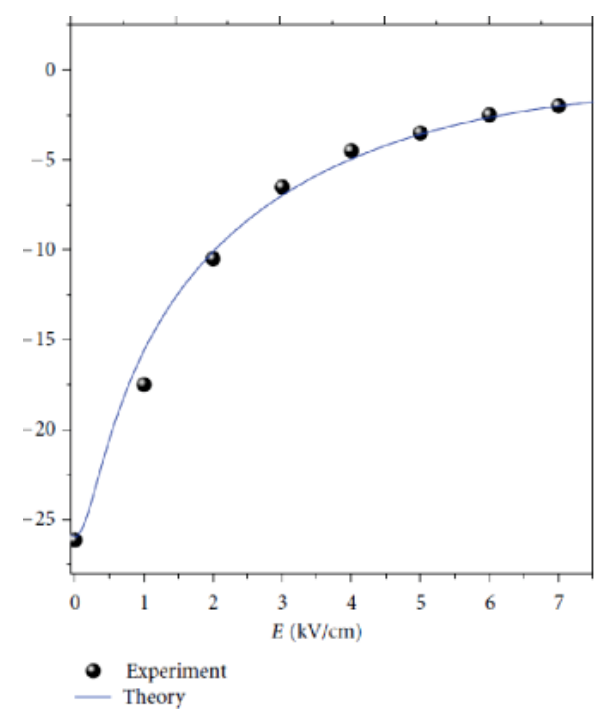

(c)

Fig. 2. (a) Design of microstrip ME attenuator and ME resonator. (b) Experimental curves of insertion loss versus frequency. YIG [111] thickness is $110 \mu \mathrm{m}$; diameter is $2.5 \mathrm{~mm}$; PMN-PT thickness is $0.5 \mathrm{~mm}$; diameter is $6 \mathrm{~mm}$; field $H=1910 \mathrm{Oe}$; field is parallel to plane of sample; central frequency is $7251 \mathrm{MHz}$. (c) Comparison of experimental and theoretical data. YIG [111] thickness is $110 \mu \mathrm{m}$; diameter is $2.5 \mathrm{~mm}$; PMN-PT thickness is $0.5 \mathrm{~mm}$; diameter is $6 \mathrm{~mm}$. Field $H=1910 \mathrm{Oe}$; field is parallel to plane of sample; average is 30 ; central frequency is $7251 \mathrm{MHz}^{29}$ 
between ferromagnetic slab and ferroelectric substrate. Achieving strong ME coupling in multiferroic heterostructures has been of paramount importance for achieving multiferroic devices with large tunability. First of all, magnetic thin film/ferroelectric slab is an improved multiferroic heterostructure for its better adhesion at the interface and magnetic thin film can be influenced by strain/stress change easily. In addition, known from Eq. (1), to further increase the ME coupling coefficient, magnetic thin film with large magnetostriction $\lambda_{S}$ and piezoelectric substrate with large piezoelectric coefficient of $d$ should be considered.

Lou et al. ${ }^{37}$ developed a FeGaB/PZN-PT (lead zinc niobate-lead titanate) bilayer multiferroic heterostructure, in which, the FeGaB alloy has high magnetostriction of $\sim 80$ ppm and relative small coercivity field of $<10$ Oe and narrow FMR linewidth of $<5$ Oe that proves the good soft and $\mathrm{RF} /$ microwave property of $\mathrm{FeGaB}$ thin films. The PZN-PT single crystal that has high piezoelectric coefficient $>3000 \mathrm{pC} / \mathrm{N}$ serves as piezoelectric substrate. By applying E-field of $0 \mathrm{kV} / \mathrm{cm}$ to $8 \mathrm{kV} / \mathrm{cm}$, a large FMR frequency (S21) switching from $1.75 \mathrm{GHz}$ to $7.57 \mathrm{GHz}(5.82 \mathrm{GHz})$ was obtained, see Fig. 3. These novel ME multiferroic heterostructures with strong ME coupling provide great opportunities for multiferroic devices.

To push the FMR tunability limitation, Liu et al. then demonstrated a Terfenol-D/PZN-PT composite bilayer structure with an effective E-field-induced magnetic anisotropy field of $3500 \mathrm{Oe}$ and a corresponding ME coefficient of $580 \mathrm{Oe} \mathrm{cm} / \mathrm{kV}^{42}$ So far, this is the highest E-field-induced ME coupling field to date, resulting in a strong tunable FMR in both amorphous and crystalline Terfenol-D films. The reason why they choose Terfenol-D alloy thin film as magnetic thin film is that it has the highest magnetostriction of $320-420 \mathrm{ppm}$ among magnetic materials. Figure 4(a) shows the E-field dependence of FMR spectrum of TerfenolD/PZN-PT. The FMR field is shifted upward by 3500 Oe as applying an E-field of $6 \mathrm{kV} / \mathrm{m}$, indicating a record-high E-field-induced FMR field change of 3500 Oe and a giant

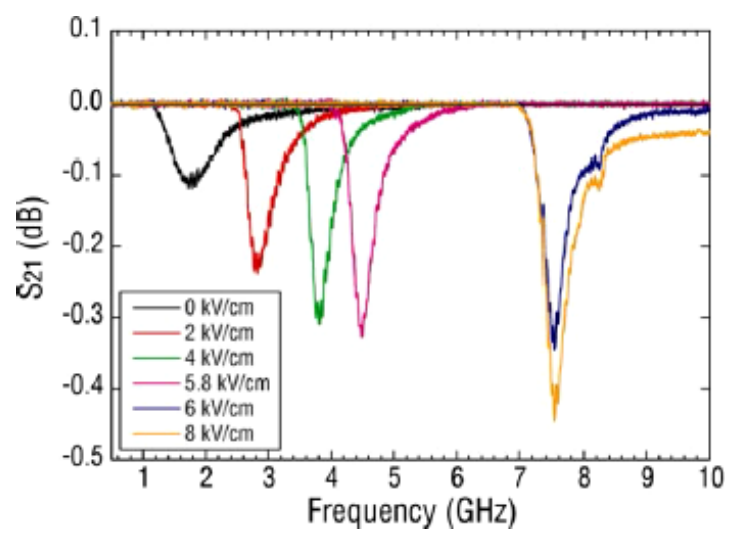

Fig. 3. (Color online) Data showing a high electric field tunable FMR frequency range from $1.75 \mathrm{GHz}$ to $7.57 \mathrm{GHz}$ at zero magnetic field and $0 \mathrm{kV} / \mathrm{cm}$ to $6 \mathrm{kV} / \mathrm{cm}$ E-field. ${ }^{37}$
ME coefficient of $580 \mathrm{Oe} \mathrm{cm} / \mathrm{kV}$. The E-field dependence of FMR field or both amorphous and polycrystalline TerfenolD/PZN-PT composites (see Fig. 4(b)) was also investigated. The resonance fields or effective magnetic fields were dramatically increased up to 3500 Oe and 2700 Oe for polycrystalline and amorphous Terfenol-D/PZN-PT, respectively.

Although giant tunability of Terfenol-D/PZN-PT was achieved, the microwave property like linewidth of Terfenol$\mathrm{D}$ is very large and that will limit its application in real devices. Moreover, the tunability of FMR frequency, instead of FMR field, should be studied. By using a new technical solution with dual E- and H-field controlling, the ME tunability dramatically enhanced FMR tunability range up to 13.1 GHz in FeGaB/PZN-PT multiferroic heterostructure, ${ }^{43}$ see Figs. 5(a) and 5(b), which will greatly satisfy engineering requirements for a variety of microwave applications. In addition, with regard to the hysteretic and reversible E-fieldinduced phase transition in single-crystal PZN-PT (011) substrates, we successfully realize a novel voltage-impulseinduced memory-type magnetization switching and FMR tuning in FeGaB/PZN-PT(011) multiferroic heterostructures. Extremely large converse $\mathrm{ME}$ coupling coefficients of $3850 \mathrm{Oe} \mathrm{cm} \mathrm{kV}^{-1}$ and $3620 \mathrm{Oe} \mathrm{cm} \mathrm{kV}^{-1}$ are observed at

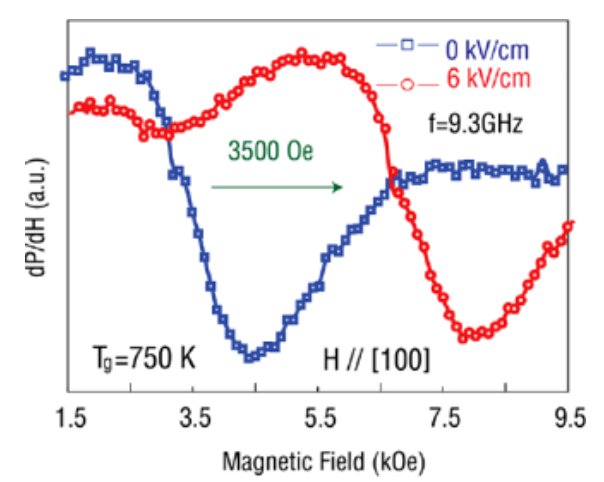

(a)

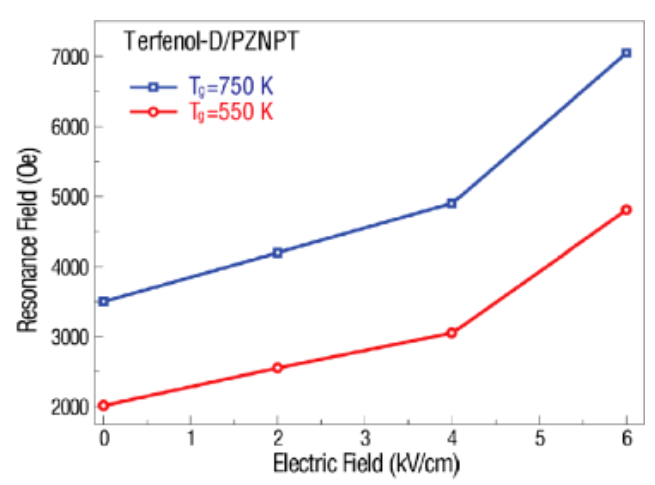

(b)

Fig. 4. (Color online) (a) FMR spectra of polycrystalline Terfenol$\mathrm{D} / \mathrm{PZN}-\mathrm{PT}$ at $E=0 \mathrm{kV} / \mathrm{cm}$ (blue) and $E=6 \mathrm{kV} / \mathrm{cm}$ (red). (b) E-field dependence of resonance field for polycrystalline (red) and amorphous (blue) Terfenol-D/PZN-PT. ${ }^{41}$ 


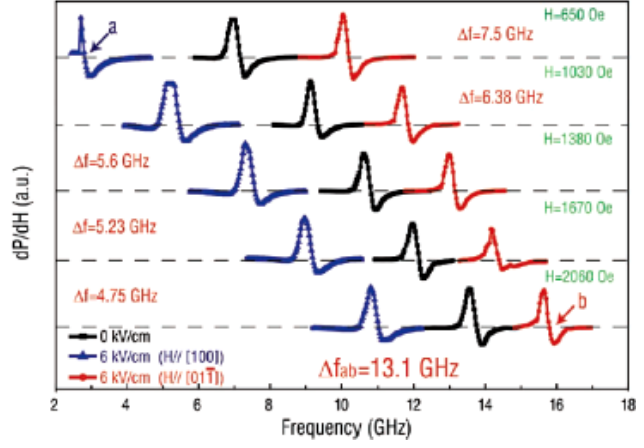

(a)

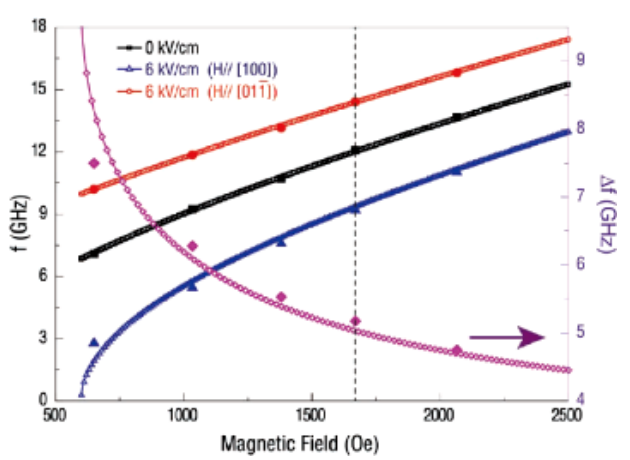

(b)

Fig. 5. (a) E-field-induced FMR frequency shift under various magnetic bias fields. (b) Comparison of theoretical simulation (solid line) and experimental results (symbols) of electric field-induced FMR change under various magnetic bias fields. ${ }^{42}$

phase transition points of $3 \mathrm{kV} \mathrm{cm}^{-1}$ and $5.8 \mathrm{kV} \mathrm{cm}^{-1}$, respectively.

Besides FMR tunability, the nonvolatile controlling use voltage impulse, instead of constant voltage to tune the FMR frequency, is of great importance of nonvolatile ME devices that has much smaller energy consumption and with strong memory effect. Liu et al. ${ }^{43}$ studied a unique ferroelastic switching pathway in (011)-oriented PMN-PT $(0.71 \mathrm{~Pb}$ $\left.\left(\mathrm{Mg}_{1 / 3} \mathrm{Nb}_{2 / 3}\right) \mathrm{O}_{3}-0.29 \mathrm{PbTiO}_{3}\right)$, see Fig. 6(a), single crystals that allows up to $90 \%$ of the polarization to rotate from an out-of-plane to a purely IP direction $\left(71^{\circ}\right.$ and $109^{\circ}$ switching), thereby producing two distinct, stable and electrically reversible lattice strain states. All FMR measurements were taken in a co-planar waveguide, see Fig. 6(b). Voltageimpulse switching between these remnant strain states is demonstrated and results in a highly energy-efficient, nonvolatile tuning of FMR field of 320 Oe (Fig. 6(c)) and FMR frequency up to $2.3 \mathrm{GHz}$ (Fig. 6(d)) in elastically coupled amorphous $\mathrm{FeCoB}$ films deposited on PMN-PT (011). A nonvolatile FMR switching of $2.3 \mathrm{GHz}$ back and forth was successfully achieved in a definite E-field range, see Figs. 6(e) and 6(f), and paves a way to nonvolatile RF/ microwave devices.

\subsection{FMR tuning in thin films multiferroic heterostructures}

In real Si substrate-based integrated RF/microwave devices, piezoelectric thin films may be applied to Si substrate for FMR tuning of magnetic thin film on top. Nevertheless, there is a fundamental challenge that the deposition temperature of conventional piezoelectric materials like PZT, PMN-PT and PZN-PT are very high $\left(>600^{\circ} \mathrm{C}\right)$ that will ruin the Si substrate. Additionally, bulk piezoelectric substrate $(0.2-0.5 \mathrm{~mm})$ requires high voltage for tuning $(>600 \mathrm{~V})$, which is too large for integrating electronics system. In short, multiferroic heterostructures with thin piezoelectric films should be considered well in multiferroic devices. Zhou et al. developed a novel $\mathrm{Fe}_{3} \mathrm{O}_{4} / \mathrm{ZnO}$ multiferroic heterostructure ${ }^{46,47}$ by spin spray low temperature fabrication method microwave $\mathrm{ME}$ interactions and magnetic tunabilities of the $\mathrm{Fe}_{3} \mathrm{O}_{4} / \mathrm{ZnO}$ multilayer were demonstrated by electrostatic field-induced IP FMR field changes at room temperature, as shown in Fig. 7(a). Here, a custom-made microwave FMR spectrometer using a planar transmission line ${ }^{48}$ was used to perform the FMR measurements of the ferrite/ferroelectric ME thin films composites at X-band $(9.3 \mathrm{GHz})$ with high sensitivity ( 1Oe). In Fig. 7(a), the bias magnetic field was applied in the $\mathrm{Fe}_{3} \mathrm{O}_{4} / \mathrm{ZnO}$ film plane with IP microwave RF field and was perpendicular to the DC bias field. A clear E-field-induced effective magnetic field $14 \mathrm{Oe}$, correspondingly, was observed between a bias voltage of $-20 \mathrm{~V}$ and $20 \mathrm{~V}$, which is indicated by the electric field-induced FMR field change, as shown in bottom right inset of Fig. 7(b). Figure 7(c) shows the FMR field dependence of applied voltage from $-30 \mathrm{~V}$ to $30 \mathrm{~V}$. The FMR field varies linearly as applied voltage switches from $-20 \mathrm{~V}$ to $20 \mathrm{~V}$, indicating the piezoelectric property of $\mathrm{ZnO}$ thin film.

\section{Voltage Control of FMR through other Mechanisms- Induced ME Coupling}

As we predicted, the piezoelectric thin film layer derived multiferroic heterostructure has limited FMR tunability due to sample clamping effect that the piezoelectric deformation energy was significant consumed in thick substrate like Si and glass. Therefore, in real $\mathrm{Si}$ substrate integrated multiferroic devices, ME coupling based on other mechanisms beyond strain/stress need to be developed. People have discovered several new ME coupling mechanisms (EM-spin wave coupling, interfacial charge, MTJ, BFO) that can control FMR properties in thin film multiferroic heterostructure without suffering from sample clamping effect. Detailed discussions are listed in each section.

\subsection{FMR tuning by EM-spin wave coupling}

Das and coworkers have recently demonstrated an YIG/ BSTO thin film multiferroic FM/FE heterostructure for 


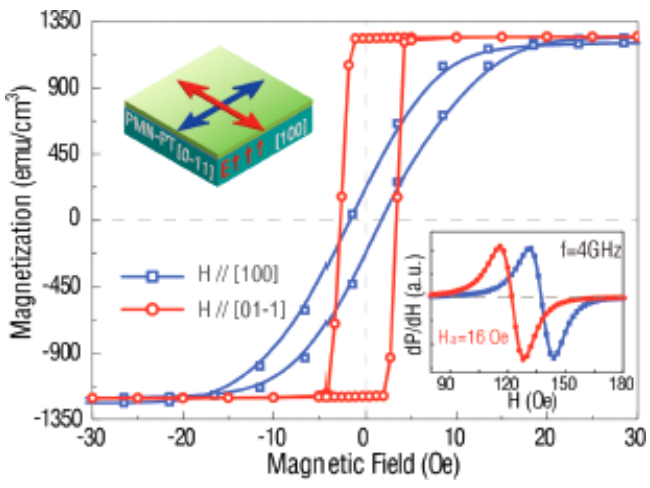

(a)

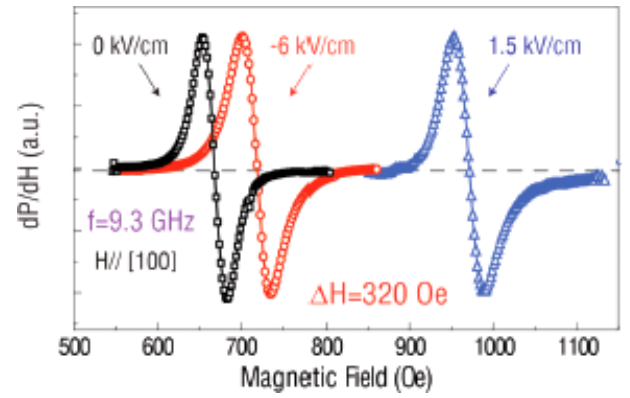

(c)

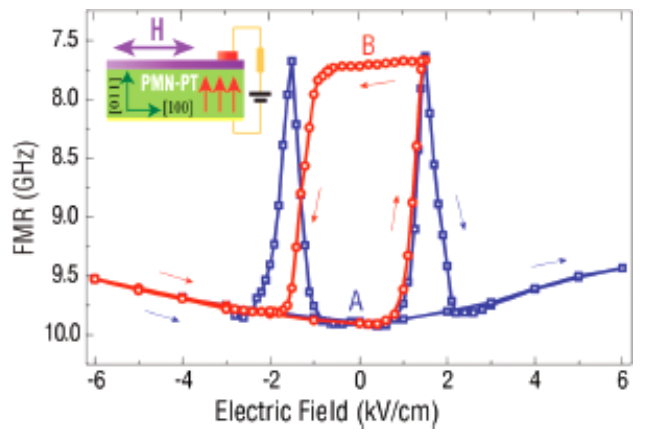

(e)

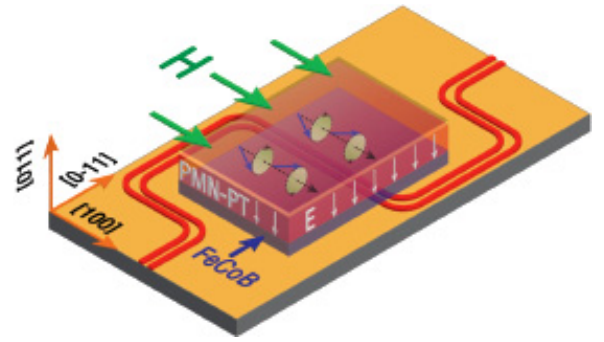

(b)

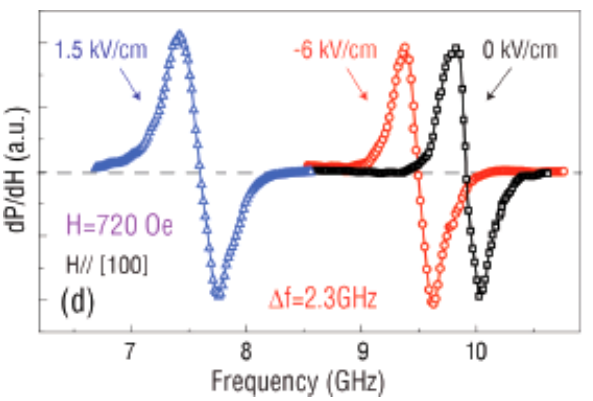

(d)

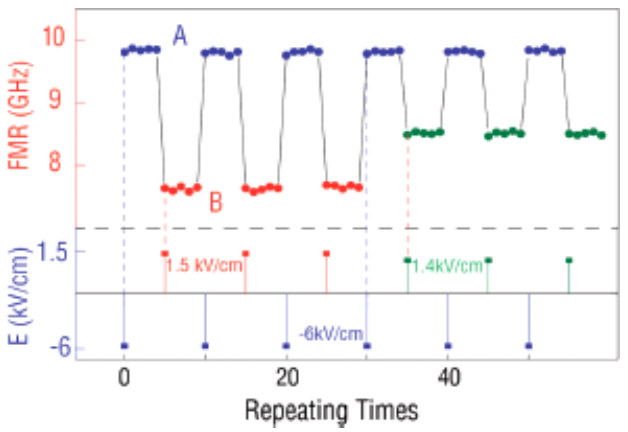

(f)

Fig. 6. (Color online) (a) IP magnetic hysteresis loops of FeCoB/PMN-PT (011). Insets are schematic (upper left) and FMR spectra (bottom right). (b) Schematic of FMR measurement for (c)-(f). The sample is laid face down on an S-shape co-planar waveguide. Magnetic fields are applied in the [100] direction and electric fields are applied along the [011] direction. (c) Electric field dependence of the FMR frequency in field sweeping mode. (d) Electric field dependence of the FMR field in frequency sweeping mode. (e) FMR frequency responses under unipolar (red) and bipolar (blue) sweeping of electric fields at room temperature. (f) Voltage-impulse-induced nonvolatile switching of FMR frequency. ${ }^{43}$

voltage tunable RF/microwave devices. ${ }^{49}$ This heterostructure consisted of a pulsed-laser-deposited (PLD) deposited textured $0.3 \mu \mathrm{m}$ YIG layer, an oriented $1 \mu \mathrm{m}$ barium strontium titanate (BSTO) layer, and embedded $50 \mathrm{~nm}$ platinum $(\mathrm{Pt})$ electrodes between the BSTO and YIG layers on a single-crystal gadolinium gallium garnet (GGG) substrate. By applying voltage of $0-25 \mathrm{~V}$ on BSTO layer, the thin film heterostructure showed an electric field tunable FMR response shift by 5 Oe through the coupling between EM wave in BSTO and spin wave in YIG, resulting in a tunability of $2 \mathrm{MHz} / \mathrm{V}$ at $9.5 \mathrm{GHz}$, as shown in Figs. 8(a) and $8(\mathrm{~b})$. In another paper, Das and coworkers also demonstrated a BaM/BSTO heterostructure, which was made of $200 \mu \mathrm{m}$ thick IP $c$-axis oriented single crystalline BaM combined with PLD BSTO films. ${ }^{48}$ A tunability of FMR frequency of $3.5 \mathrm{MHz} / \mathrm{V}$ has been observed at $60 \mathrm{GHz}$ by applying bias voltages in the range of $0-6 \mathrm{~V}$. The fundamental limitation of EM-spin wave coupling tuning of FMR frequency is that the tunability is too small for real applications. 


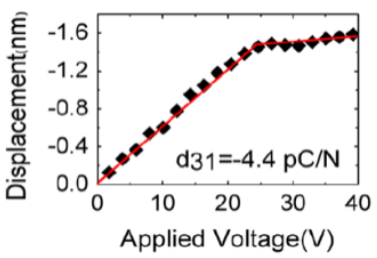

(a)

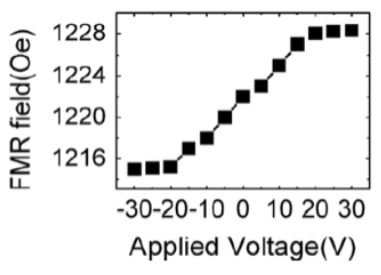

(c)

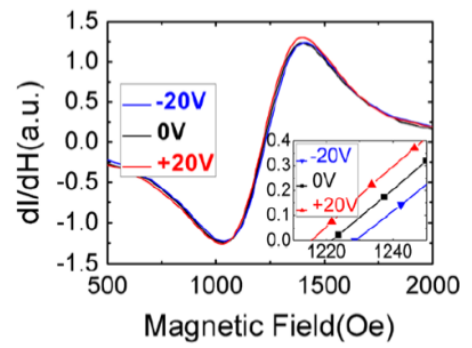

(b)

Fig. 7. (a) Piezoelectric coefficient measurements of $\mathrm{ZnO}$ thin film. (b) Electric field dependence of the IP field-sweep FMR spectra of the $\mathrm{Fe}_{3} \mathrm{O}_{4} / \mathrm{ZnO} \mathrm{ME}$ heterostructure measured at $9.3 \mathrm{GHz}$. The zero cross part was enlarged to demonstrate a clear ME coupling shift at bottom right inset; (c) X-band IP FMR field of the $\mathrm{Fe}_{3} \mathrm{O}_{4} / \mathrm{ZnO}$ heterostructure measured at varying applied voltages across the $\mathrm{ZnO}$ layer. $^{47}$

\subsection{FMR tuning by interfacial charge-induced ME coupling}

Strong ME coupling has been demonstrated in magnetic/ dielectric or magnetic/ferroelectric thin film heterostructures through a voltage controllable magnetic surface anisotropy mediated by spin polarized charge. ${ }^{51-59}$ The charge accumulation at ultrathin magnetic/dielectric or ferroelectric interface will change the spin-orbit coupling of magnetic layer ${ }^{51-57}$ and then influence the surface anisotropy of magnetic layer, resulting in FMR frequency change. Nan et al. ${ }^{58}$ studied the $\mathrm{NiFe}(\sim 1 \mathrm{~nm}) / \mathrm{PMN}-\mathrm{PT}$ bilayer heterostructure where the strain and charge co-mediated ME coupling are expected, which could lead to significantly enhanced ME coupling. It is however challenging to observe the combined strain charge mediated ME coupling, and difficult to quantitatively distinguish these two ME coupling mechanisms. Nan et al. ${ }^{58}$ demonstrated in this work that, by inserting a thin

$\mathrm{Cu}$ layer between NiFe and PMN-PT, the charge effect can be excluded with only strain-mediated ME coupling left. A 202 Oe FMR field shift was shown in only strain-mediated ME coupling and a total 357 Oe FMR field change was demonstrated in strain and charge co-existed NiFe/PMN-PT multiferroic heterostructure. By distinguishing the ME coupling mechanisms, a pure surface charge modification of magnetism shows a strong correlation to polarization of PMN-PT, see Fig. 9. A nonvolatile effective H-field change of 104 Oe was observed at zero electric field originating from different remnant polarization states of PMN-PT. The strain and charge co-mediated ME coupling in ultra-thin magnetic/ ferroelectric heterostructures could lead to power efficient and nonvolatile ME devices with enhanced ME coupling.

After distinguishing the charge mediated ME coupling strength, Zhou et $a l .{ }^{59}$ then investigated the correlation between charge mediated ME coupling strength and thickness of NiFe ultrathin magnetic film in order to maximize the performance of charge-mediated ME devices. Precise quantification of the ME coupling strength in surface chargeinduced $\mathrm{ME}$ effect was studied in $\mathrm{NiFe} / \mathrm{SrTiO}_{3}$ thin film heterostructures with different ultra-thin $\mathrm{NiFe}$ thicknesses through voltage-induced FMR measurements, as shown in Fig. 10(a). As demonstrated in Fig.10(b), the voltage-induced FMR field shifts in these $\mathrm{NiFe} / \mathrm{SrTiO}_{3}$ thin films heterostructures showed a maximum value of $65 \mathrm{Oe}$ at an intermediate $\mathrm{NiFe}$ layer thickness of $1.2 \mathrm{~nm}$, which was interpreted based on the thin film growth model at the low thicknesses and on the charge screening effect at large thicknesses. Figure 10(c) shows the angular dependence of FMR switching under varied E-field, the isotropic FMR change implies the charge effect-induced out-of-plane surface anisotropy change of magnetic thin films. ${ }^{51-57}$ By optimizing the magnetic thin film thickness, the ME devices with stronger FMR tunability can be achieved.

\subsection{FMR tuning in MTJ}

Voltage control of magnetic anisotropy will lead a way to control magnetoresistance (MR) by applying a DC voltage

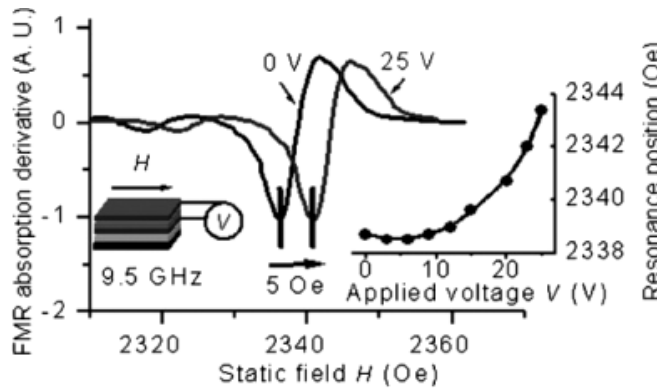

(a)

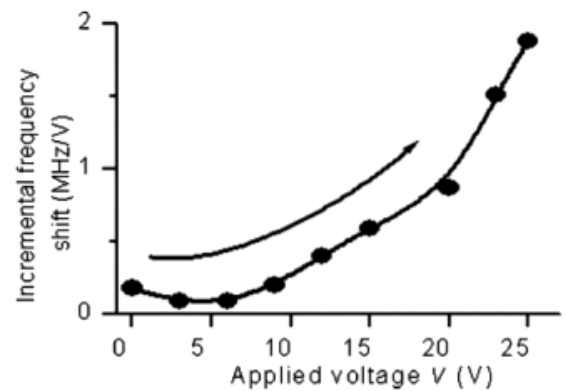

(b)

Fig. 8. Electric field tuning of the FMR response. (a) The FMR absorption derivative versus IP static field $\mathrm{H}$ spectra at $9.5 \mathrm{GHz}$ for $0 \mathrm{~V}$ and $25 \mathrm{~V}$ applied voltages, as indicated. Right inset shows. The FMR resonance position as a function of the applied voltage. (b) Incremental frequency shift versus applied voltage. ${ }^{48}$ 


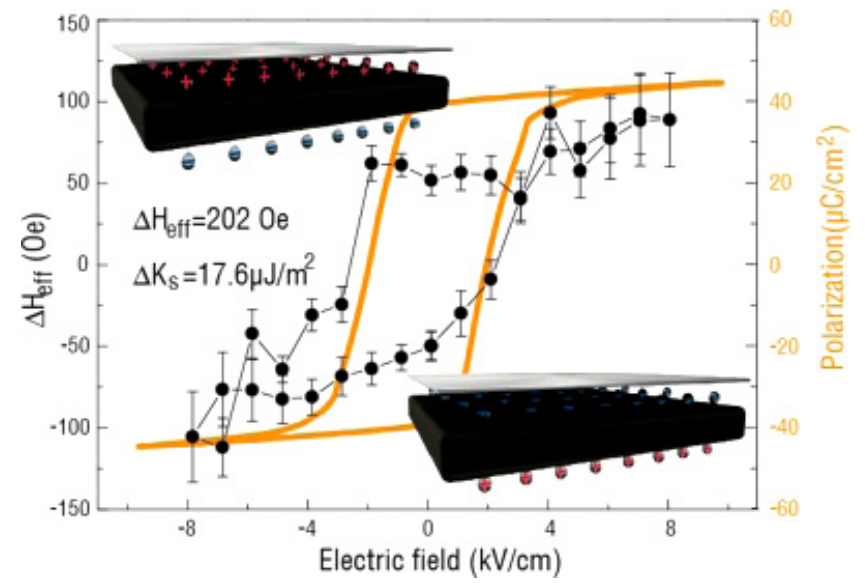

Fig. 9. (Color online) The change of the effective magnetic field upon the applied electric field induced by pure screening charge effect in NiFe/PMN-PT (black) and $P(E)$ loop of PMN-PT (orange). Insets show the schematics of the positive (up) and negative (down) screen charge on the NiFe interface. ${ }^{58}$

across the isolating layer like $\mathrm{MgO} .{ }^{60,61}$ As a consequence, a small voltage bias can reduce 1-2 order of current density for spin transfer torque (STT) that switches the magnetization of free layer in MTJ structure, see Fig. 11(a). Nozaki et al. revealed that by the FMR dynamics of ultrathin CoFe magnetic layer can be excited by using a radiofrequency (RF) voltage bias onto isolating layer at room temperature, see Fig. 11(b), and this phenomenon could enable more energy-efficient spintronic devices and related technologies compared to conventional RF magnetic fields or injection of spin-polarized current control of FMR dynamics. ${ }^{60,61}$ Figures 11(c) and 11(d) show the FMR dynamics in magnetic layer induced by RF voltage bias under different DC magnetic bias. This technique provides a low-power, highly localized and coherent means to manipulate electron spin dynamics.

\subsection{FMR tuning in CoFe/BFO system}

BFO is a very interesting multiferroic material system that has both ferroelectricity and antiferromagnetism coexistence in each crystal lattice. ${ }^{62-73}$ Ferroelectric polarization and antiferromagnetic canted moment in BFO system are coupled through Dzyaloshinskii-Moriya (DM) interaction and the antiferromagnetic vector, therefore, can be manipulated by switching the polarization of BFO by E-field. Lots of researches have been devoted to realized nonvolatile control of $180^{\circ}$ magnetization switching in exchange coupled CoFe/ BFO thin-film heterostructures, in which, the moment of $\mathrm{CoFe}$ layer is coupled to the antiferromagnetic canted moment in BFO layer. Heron et al. realized $180^{\circ}$ magnetization switching in $\mathrm{CoFe} / \mathrm{BFO}$ multiferroic heterostructure by MR measurements ${ }^{71,72}$ in previous research. Recently, Zhou et al. $^{73}$ studied the FMR dependence of CoFe/BFO by using high sensitive ESR system. Figure 12(a) shows the schematic

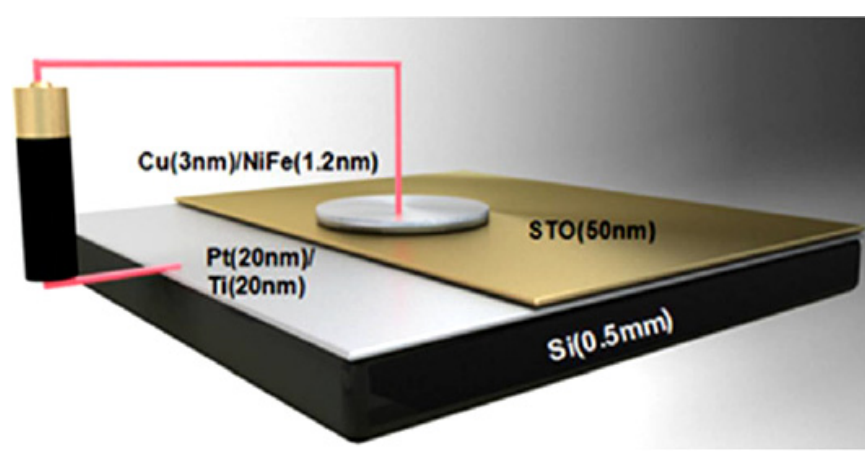

(a)

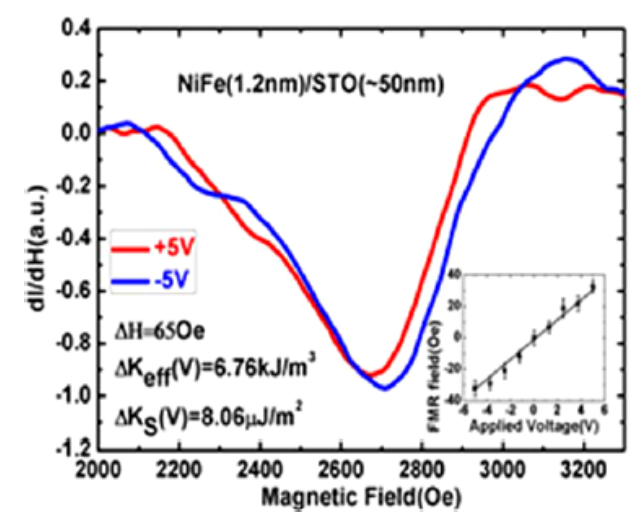

(b)

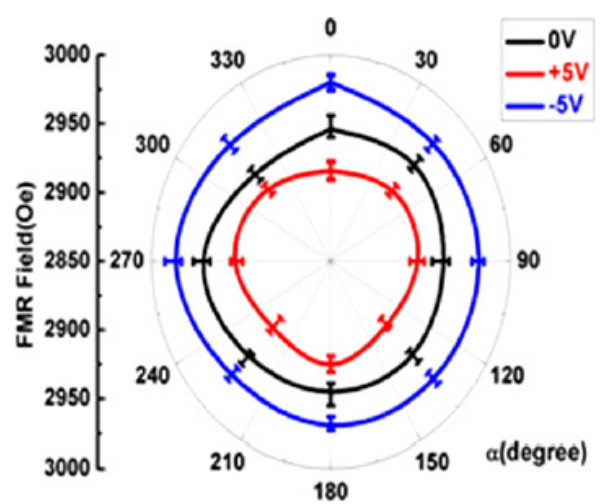

(c)

Fig. 10. (a) Schematic of the sample used for a voltage-induced FMR field change. A schematic of multilayer structure of $\mathrm{Cu} / \mathrm{NiFe} /$ $\mathrm{STO} / \mathrm{Pt} / \mathrm{Si}$. The magnetic field was applied perpendicular to the film plane for FMR measurements. (b) Voltage dependence of the IP field-sweep FMR spectra of the NiFe/STO multiferroic heterostructure measured at $9.5 \mathrm{GHz}$. The zero cross part was enlarged to demonstrate a clear ME coupling shift at bottom left inset. (c) Angular dependence of FMR field measurements in NiFe $(1.2 \mathrm{~nm}) / \mathrm{STO}$ $(50 \mathrm{~nm})$ under varying voltages: the positive (up) and negative (down) screen charge on the NiFe interface. ${ }^{59}$

of $\mathrm{CoFe} / \mathrm{BFO}$ bilayer structure with $0.4 \mathrm{~mm}$ diameter $\mathrm{CoFe}$ spot on BFO thin layer and the voltage impulses were applied onto BFO layer. Figure 12(b) shows the FMR spectra under varied E-field and a significant nonvolatile FMR shift was 


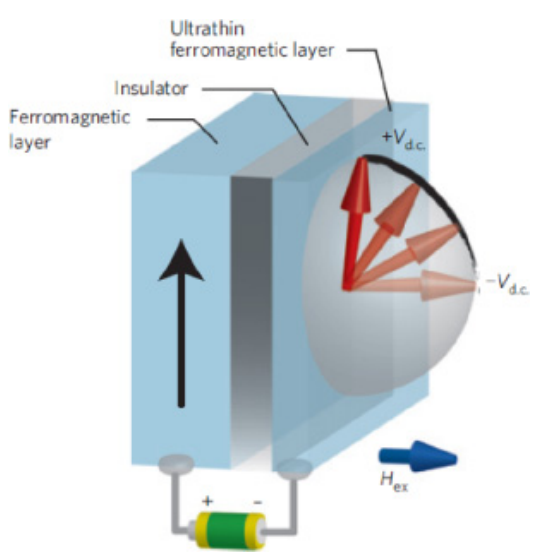

(a)

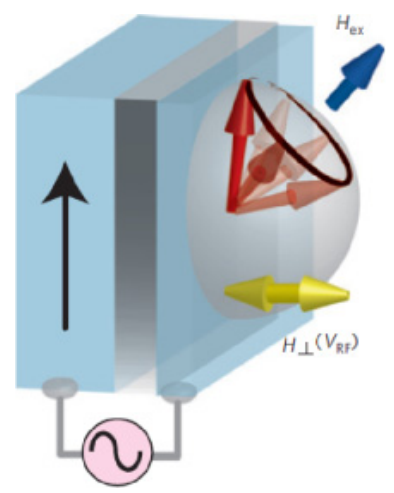

(b)

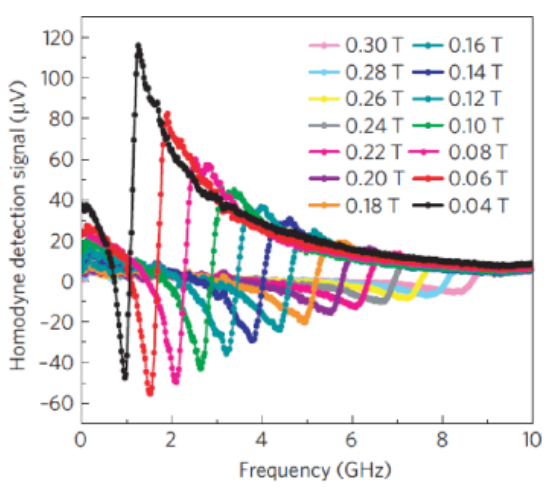

(c)

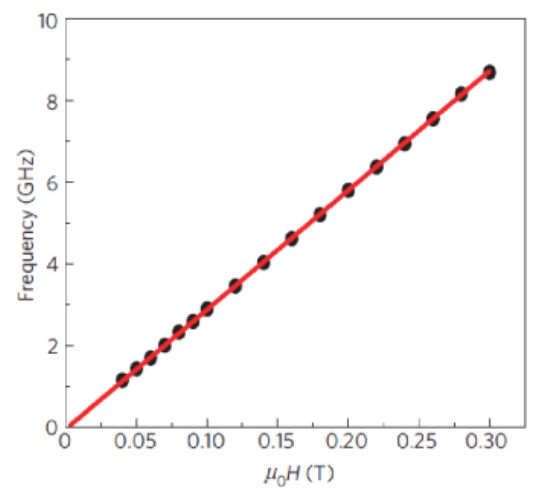

(d)

Fig. 11. (Color online) E-field-induced FMR in a magnetic tunnel junction with an ultrathin ferromagnetic layer. (a) Application of a DC voltage can switch the magnetic easy axis between the IP and out-of-plane directions. (b) Application of a RF voltage can excite the FMR dynamics under a static external magnetic field. The yellow arrow represents the RF effective field change originating from the anisotropy control. (c) External-magnetic-field dependence of homodyne detection signals measured under the fixed RF power of 32 Wand elevation angle of $55^{\circ}$. The external-magnetic-field strength was varied from $0.04 \mathrm{~T}$ to $0.3 \mathrm{~T}$. (d) Resonant frequency as a function of the external magnetic field. The results are modeled well (red line) by the modified Kittel formula. ${ }^{61}$

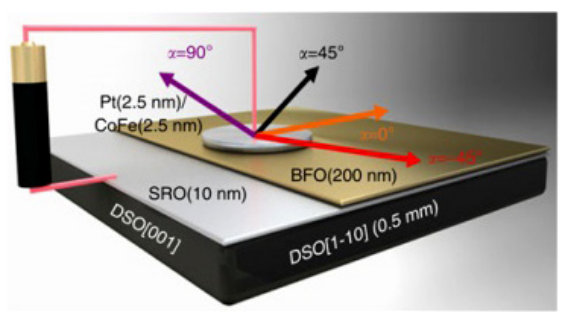

(a)

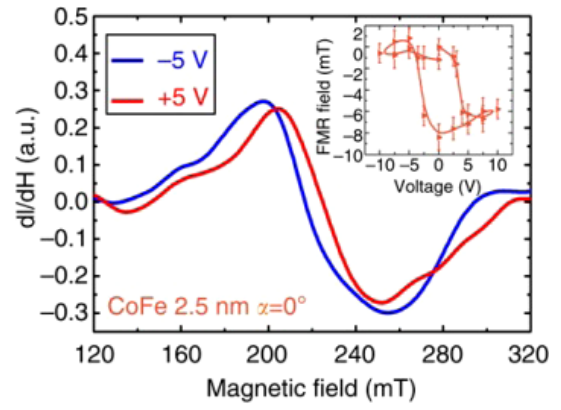

(b)

Fig. 12. FMR measurements of CoFe/BFO multiferroic heterostructure. (a) Schematic of CoFe $(2.5 \mathrm{~nm}) / \mathrm{BFO}(200 \mathrm{~nm}) \mathrm{multiferroic}$ heterostructure for FMR measurements at varying angles between the magnetic field and the easy axis. (b) FMR spectra of CoFe/BFO multiferroic heterostructure measured at open circuit after applying $+5 \mathrm{~V}$ and $-5 \mathrm{~V}$. Upper right inset is the relative FMR field dependence on applied voltage. (c) Relative FMR field dependence after applied voltage pulses along different orientations of the magnetic field. (d) Relative FMR field versus applied voltage pulses after 50 and 100 cycles of a $10-\mathrm{V}$ sinusoidal voltage. ${ }^{73}$ 


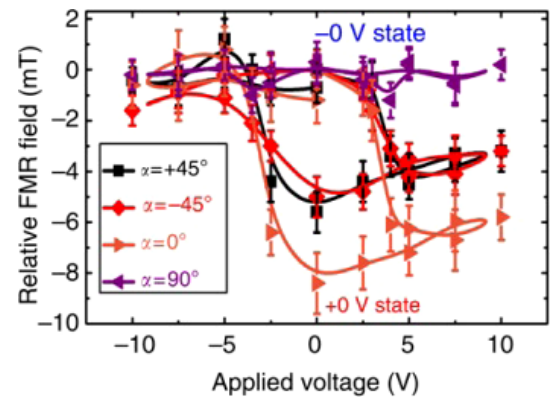

(c)

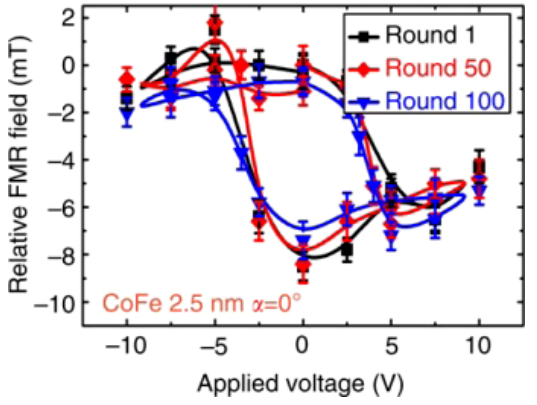

(d)

Fig. 12. (Continued)

demonstrated. As summarized in Fig. 12(c), the E-field dependence of FMR fields along different magnetic field orientations was demonstrated and that proves a unidirectional magnetic anisotropy change by exchange coupling of $\mathrm{CoFe}$ and BFO. The FMR dependence of E-field is similar to a typical polarization-electric field hysteresis loop which indicates the strong correlation between ferroelectric property and FMR properties. At last, Fig. 12(d) gives a robust FMR switching after $>100$ round testing. This progress constitutes an important step towards robust repeatable and nonvolatile voltage-induced $180^{\circ}$ magnetization switching in thin-film multiferroic heterostructures and tunable $\mathrm{RF} /$ microwave devices.

\section{Conclusion}

In summary, we introduce voltage control of FMR in multiferroic heterostructures. Firstly, conventional strain/stress ME coupling induced FMR change in different multiferroic heterostructures, from bulk/bulk magnetic/ferroelectric heterostructures to thin film/thin film heterostructures, were systematically studied. Nevertheless, sample clamping effect that reduced ME coupling strength limited its real applications. Secondly, to overcome the limitation of strain/stressinduced ME coupling for FMR tuning, other mechanisms like EM-spin wave coupling, interfacial charge and exchange coupling in BFO systems were discovered and investigated. Finally, as a unique characterization method, FMR measurement is a widely used technology to precisely determine the electric field-induced small effective magnetic anisotropy change in multiferroic system with few magnetic moments. The voltage control of FMR in multiferroics provide a path toward next generation small, fast, energy efficient voltage tuning military/civilian $\mathrm{RF} /$ microwave devices.

\section{Acknowledgments}

This work is supported by the Natural Science Foundation of China (Grant Nos. 51472199, 11534015), the National 111
Project of China (B14040), the Beijing Institute of Technology Research Fund Program for Young Scholars (Grant No. 3050012261527), W.M. Keck Foundation and National Science Foundation. Dr. Ming Liu was supported by China Recruitment Program for Young Professionals.

\section{References}

${ }^{1}$ W. Eerenstein, N. D. Mathur and J. F. Scott, Multiferroic and magnetoelectric materials, Nature 442, 759 (2006).

${ }^{2}$ M. Fiebig, Multiferroics: Progress and prospects, J. Phys. D: Appl. Phys. 38, R123 (2005).

${ }^{3}$ R. Ramesh and N. A. Spaldin, Multiferroics: Progress and prospects in thin films, Nat. Mater. 6, 21 (2007).

${ }^{4}$ C. W. Nan, M. I. Bichurin, S. X. Dong, D. Viehland and G. Srinivasan, Magnetoelectric multiferroic composites, J. Appl. Phys. 103, 031101 (2008).

${ }^{5}$ C. A. F. Vaz, J. Holfman, C. H. Ahn and R. Ramesh, Magnetoelectric coupling effects in multiferroic complex oxide composite structures, Adv. Mater. 22, 2900 (2010).

${ }^{6}$ G. Srinivasan, Magnetoelectric composites, Ann. Rev. Mater. Res. 40, 153 (2010).

${ }^{7}$ L. W. Martin, Y. H. Chu and R. Ramesh, Advances in the growth and characterization of magnetic, ferroelectric, and multiferroic oxide thin films, Mater. Sci. Eng. R 68, 89 (2010).

${ }^{8}$ J. Ma, J. Hu, Z. Li and C. W. Nan, Recent progress in multiferroic magnetoelectric composites: From bulk to thin films, Adv. Mater. 23, 1062 (2011).

${ }^{9}$ M. Liu and N. X. Sun, Voltage control of magnetism in multiferroic heterostructures, Phil. Trans. R. Soc. A 372, 20120439 (2014).

${ }^{10}$ B. Lax and K. J. Button, Microwave Ferrites and Ferrimagnetics (McGraw-Hill, New York, 1962).

${ }^{11}$ J. D. Adam, L. E. Davis, G. F. Dionne, E. F. Schloemann and S. N. Stitzer, Ferrite devices and materials, IEEE Trans. Microw. Theor. Tech. 50, 721 (2002).

${ }^{12}$ G. M. Yang, X. Xing, A. Daigle, O. Obi, M. Liu, J. Lou, S. Stoute, K. Naishadham and N. X. Sun, Planar annular ring antennas with multilayer self-biased NiCo-ferrite films loading, IEEE Trans. Antennas Propag. 58, 648 (2010).

${ }^{13}$ G. M. Yang, X. Xing, A. Daigle, O. Obi, M. Liu, S. Stoute, K. Naishadham and N. X. Sun, Loading effects of self-biased 
magnetic films on patch antennas with substrate/superstrate sandwich structure, IET Microw., Antennas Propag. 4, 1172 (2010).

${ }^{14}$ G. M. Yang, X. Xing, A. Daigle, M. Liu, O. Obi, S. Stoute, K. Naishadham and N. X. Sun, Tunable miniaturized patch antennas with self-biased multilayer magnetic films, IEEE Trans. Antennas Propag. 57, 2190 (2009).

${ }^{15}$ Y. Gao, S. Zare, X. Yang, T. X. Nan, Z. Y. Zhou, M. Onabajo, M. Liu, A. Aronow, K. Mahalingam, B. M. Howe, G. J. Brown and N. X. Sun, Significantly enhanced inductance and quality factor of $\mathrm{Ghz}$ integrated magnetic solenoid inductors with $\mathrm{Fegab} / \mathrm{Al}_{2} \mathrm{O}_{3}$ multilayer films, IEEE Trans. Electron Devices 61, 1470 (2014).

${ }^{16}$ Y. Gao, S. Zare, X. Yang, T. X. Nan, Z. Y. Zhou, M. Onabajo, K. P. O'Brien, U. Jalan, M. EI-tatani, P. Fisher, M. Liu, A. Aronow, K. Mahalingam, B. M. Howe, G. J. Brown and N. X. Sun, High Q integrated $\mathrm{GHz}$ magnetic transformers with $\mathrm{FeGaB} / \mathrm{Al}_{2} \mathrm{O}_{3}$ multilayer films for RFIC applications, J. Appl. Phys. 115, 17E714 (2014).

${ }^{17}$ X. Xing, M. Liu, S. Li, O. Obi, J. Lou, Z. Zhou, B. Chen and N. X. Sun, RF magnetic properties of $\mathrm{FeCoB} / \mathrm{Al}_{2} \mathrm{O}_{3} / \mathrm{FeCoB}$ structure with varied $\mathrm{Al}_{2} \mathrm{O}_{3}$ thickness, IEEE Trans. Magn. 47, 3104 (2011)

${ }^{18}$ X. Yang, J. Wu, Y. Gao, T. Nan, Z. Zhou, S. Beguhn, M. Liu and N. X. Sun, Compact and low loss phase shifter with low bias field using partially magnetized ferrite, IEEE Trans. Magn. 49, 3882 (2013).

${ }^{19}$ X. Yang, Y. Gao, J. Wu, S. Beguhn, T. Nan, Z. Zhou, M. Liu and N. X. Sun, Dual H- and E-Field tunable multiferroic bandpass filter at $\mathrm{Ku}$ Band Using Partially magnetized spinel ferrites, IEEE Trans. Magn. 49, 5485 (2013).

${ }^{20}$ X. Yang, J. Wu, S. Beguhn, T. Nan, Y. Gao, Z. Zhou and N. X. Sun, Tunable bandpass filter using partially magnetized ferrites with high power handling capability, IEEE Microw. Wireless Compon. Lett. 23, 184 (2013).

${ }^{21}$ X. Yang, J. Wu, J. Lou, X. Xing, D. E. Oates, G. F. Dionne and N. X. Sun, Compact tunable bandpass filter on YIG substrate, Electron. Lett. 48, 17 (2012).

${ }^{22}$ T. X. Nan, Y. Hui, M. Rinaldi and N. X. Sun, Self-biased $215 \mathrm{MHz}$ magnetoelectric MEMS resonator for ultra-sensitive DC magnetic field detection, Sci. Rep. 3, 1985 (2013).

${ }^{23}$ J. Zhai, Z. Xing, S. Dong, J. Li and D. Viehland, Detection of pico-Tesla magnetic fields using magneto-electric sensors at room temperature, Appl. Phys. Lett. 88, 062510 (2006).

${ }^{24}$ S. X. Dong, J. Y. Zhai, J. F. Li and D. Viehland, Multimodal system for harvesting magnetic and mechanical energy, Appl. Phys. Lett. 93, 103511 (2008).

${ }^{25}$ A. S. Tatarenko, V. Gheevarughese and G. Srinivasan, Magnetoelectric microwave bandpass filter, Electron. Lett. 42, 540 (2006).

${ }^{26}$ C. Pettiford, S. Dasgupta, J. Lou, S. D. Yoon and N. X. Sun, Compact and low loss phase shifter with low bias field using partially magnetized ferrite, IEEE Trans. Magn. 43, 3343 (2007).

${ }^{27}$ Y. K. Fetisov and G. Srinivasan, Electric field tuning characteristics of a ferrite-piezoelectric microwave resonator, Appl. Phys. Lett. 88, 143503 (2006).

${ }^{28}$ A. S. Tatarenko, G. Srinivasan and M. I. Bichurin, Electrically tunable ferrite-ferroelectric microwave delaylines, Appl. Phys. Lett. 88, 183507 (2006).

${ }^{29}$ A. S. Tatarenko and M. I. Bichurin, Microwave magnetoelectric devices, Adv. Condens. Matter Phys. 2012, 286562 (2012).
${ }^{30}$ S. X. Dong, J. F. Li and D. Viehland, Ultra-high magnetic field sensitivity in laminates of TERFENOL-D and PMN-PT crystals, Appl. Phys. Lett. 83, 2265 (2003).

${ }^{31}$ M. Li, Z. Zhou, M. Liu, J. Lou, D. E. Oates, G. F. Dionne, M. L. Wang and N. X. Sun, Novel NiZnAl-ferrites and strong magnetoelectric coupling in NiZnAl-ferrite/PZT multiferroic heterostructures, J. Phys. D: Appl. Phys. 46, 275001 (2013).

${ }^{32}$ T. Wu, A. Bur, P. Zhao, K. P. Mohanchandra, K. Wong, K. L. Wang, C. S. Lynch and G. P. Carman, Giant electric-field-induced reversible and permanent magnetization reorientation on magnetoelectric $\mathrm{Ni} /(011)\left[\mathrm{Pb}\left(\mathrm{Mg}_{1 / 3} \mathrm{Nb}_{2 / 3}\right) \mathrm{O}_{3}\right]_{(1-x)}-\left[\mathrm{PbTiO}_{3}\right]_{x}$ heterostructure, Appl. Phys. Lett. 98, 012504 (2011).

${ }^{33}$ M. Liu, J. Lou, S. Li, S. Rand, O. Obi, X. Xing and N. X. Sun, Electric field modulation of magnetoresistance in multiferroic heterostructures for ultralow power electronics, Appl. Phys. Lett. 98, 222509 (2011).

${ }^{34}$ M. Liu, J. Lou and N. X. Sun, E-field control of exchange bias and deterministic magnetization switching in AFM/FM/FE multiferroic heterostructures, Adv. Funct. Mater. 21, 2593 (2011).

${ }^{35}$ J. M. Hu, Z. Li, L. Q. Chen and C. W. Nan, High-density magnetoresistive random access memory operating at ultralow voltage at room temperature, Nat. Commun. 2, 553 (2011).

${ }^{36}$ T. X. Nan, Z. Zhou, J. Lou, M. Liu, X. Yang, Y. Gao, S. Rand and N. X. Sun, Voltage impulse induced bistable magnetization switching in multiferroic heterostructures, Appl. Phys. Lett. 100, 132409 (2012).

${ }^{37}$ J. Lou, M. Liu, D. Reed, Y. H. Ren and N. X. Sun, Giant electric field tuning of magnetism in novel multiferroic FeGaB/lead zinc niobate lead titanate heterostructures, Adv. Mater. 21, 4711 (2009).

${ }^{38}$ S. Li, H. Du, Q. Xue, X. Gao, Y. Zhang, W. Shao, T. Nan, Z. Zhou and N. X. Sun, Large E-field tunability of microwave ferromagnetic properties in $\mathrm{Fe}_{59.3} \mathrm{Co}_{28.0} \mathrm{Hf}_{12.7} / \mathrm{PZN}-\mathrm{PT}$ multiferroic composites, J. Appl. Phys. 115, 17C723 (2014).

${ }^{39}$ S. Li, H. Du, Y. Zhang, Q. Xue, X. Gao, W. Shao, Z. Zhou, T. Nan and N. X. Sun, Quasi magnetic isotropy and microwave performance of $\mathrm{FeCoB}$ multilayer laminated by uniaxial anisotropic layers, J. Appl. Phys. 115, 17A310 (2014).

${ }^{40}$ Z. Zhou, X. Y. Zhang, T. F. Xie, T. X. Nan, Y. Gao, X. Yang, X. Y. He, P. S. Qiu, N. X. Sun and D. Z. Sun, Strong non-volatile voltage tuning of ferromagnetic resonance with bistable magnetization switching in magnetic/antiferroelectric FeGaB/PSZT heterostructures, Appl. Phys. Lett. 104, 012905 (2014).

${ }^{41}$ M. Liu, S. Li, Z. Zhou, S. Beguhn, J. Lou, F. Xu, T. J. Lu and N. X. Sun, Electrically induced enormous magnetic anisotropy in Terfenol-D/lead zinc niobate-lead titanate multiferroic heterostructures, J. Appl. Phys. 112, 063917 (2012).

${ }^{42}$ M. Liu, Z. Zhou, T. Nan, B. M. Howe, G. J. Brown and N. X. Sun, Voltage tuning of ferromagnetic resonance with bistable magnetization switching in energy-efficient magnetoelectric composites, Adv. Mater. 25, 1435 (2013).

${ }^{43}$ M. Liu, B. M. Howe, L. Grazulis, K. Mahalingam, T. X. Nan, N. X. Sun and G. J. Brown, Voltage-impulse-induced non-volatile ferroelastic switching of ferromagnetic resonance for reconfigurable magnetoelectric microwave devices, Adv. Mater. 25, 4886 (2013).

${ }^{44}$ Z. Zhou, S. Beguhn, J. Lou, S. Rand, M. Li, X. Yang, S. D. Li, M. Liu and N. X. Sun, Low moment NiCr radio frequency magnetic films for multiferroic heterostructures with strong magnetoelectric coupling, J. Appl. Phys. 111, 103915 (2012). 
${ }^{45}$ N. Li, M. Liu, Z. Zhou, N. X. Sun, D. V. B. Murthy, G. Srinivasan, T. M. Klein, V. M. Petrov and A. Gupta, Electrostatic tuning of ferromagnetic resonance and magnetoelectric interactions in ferrite-piezoelectric heterostructures grown by chemical vapor deposition, Appl. Phys. Lett. 99, 192502 (2011).

${ }^{46}$ Z. Zhou, O. Obi, S. Beghun, T. X. Nan, S. Stoute, M. Liu, J. Lou, X. Yang, Y. Gao, M. Li, X. Xing, N. X. Sun, J. Warzywoda, A. Sacco Jr., T. Guo and C. W. Nan, Growth behaviors and characteristics of low temperature spin-sprayed $\mathrm{ZnO}$ and Al-doped $\mathrm{ZnO}$ microstructures, J. Mater. Sci.: Mater. Electron. 12, 1056 (2013).

${ }^{47}$ Z. Zhou, O. Obi, T. X. Nan, S. Beguhn, J. Lou, X. Yang, Y. Gao, M. Li, S. Rand, H. Lin, N. X. Sun, G. Esteves, K. Nittala, J. L. Jones, K. Mahalingam, M. Liu and G. J. Brown, Low-temperature spin spray deposited ferrite/piezoelectric thin film magnetoelectric heterostructures with strong magnetoelectric coupling, J. Mater. Sci. Mater. Electron. 25(3), 1188 (2014).

${ }^{48}$ S. Beguhn, Z. Zhou, S. Rand, X. Yang, J. Lou and N. X. Sun, A new highly sensitive broadband ferromagnetic resonance measurement system with lock-in detection, J. Appl. Phys. 111, 07A503 (2012).

${ }^{49}$ J. Das, Y.-Y. Song, N. Mo, P. Krivosik and C. E. Patton, Electricfield-tunable low loss multiferroic ferrimagnetic-ferroelectric heterostructures, Adv. Mater. 21, 2045 (2009).

${ }^{50}$ Y. Y. Song, J. Das, P. Krivosik, H.-K. Seo and M. Wu, Electric tuning of ferromagnetic resonances in hexagonal-barium-ferrite/ barium-strontium-titanate heterostructures, IEEE Magn. Lett. 1, 2500204 (2010).

${ }^{51}$ C. G. Duan, S. S. Jaswal and E. Y. Tsymbal, Predicted magnetoelectric effect in $\mathrm{Fe} / \mathrm{BaTiO}_{3}$ multilayers: Ferroelectric control of magnetism, Phys. Rev. Lett. 97, 047201 (2006).

${ }^{52} \mathrm{Z}$. Li et al., Thickness-dependent converse magnetoelectric coupling in bi-layered Ni/PZT thin films, J. Appl. Phys. 111, 033918 (2012).

${ }^{53}$ L. Shu et al., Thickness-dependent voltage-modulated magnetism in multiferroic heterostructures. Appl. Phys. Lett. 100, 022405 (2012).

${ }^{54} \mathrm{~T}$. Maruyama et al., Large voltage-induced magnetic anisotropy change in a few atomic layers of iron, Nat. Nanotechnol. 4, 158 (2009).

${ }^{55}$ Y. Shiota et al., Voltage-assisted magnetization switching in ultrathin $\mathrm{Fe}_{80} \mathrm{Co}_{20}$ alloy layers, Appl. Phys. Exp. 2, 063001 (2009).

${ }^{56}$ C. G. Duan et al., Tailoring magnetic anisotropy at the ferromagnetic/ferroelectric interface, Appl. Phys. Lett. 92, 122905 (2008).
${ }^{57}$ Z. Zhou, B. Howe, M. Liu, T. Nan, X. Chen, K. Mahalingam, N. X. Sun and G. J. Brown, Interfacial charge-mediated nonvolatile magnetoelectric coupling in $\mathrm{Co}_{0.3} \mathrm{Fe}_{0.7} / \mathrm{Ba}_{0.6} \mathrm{Sr}_{0.4} \mathrm{TiO}_{3} / \mathrm{Nb}$ : $\mathrm{SrTiO}_{3}$ multiferroic heterostructures, Sci. Rep. 5, 7740 (2015).

${ }^{58}$ T. Nan et al., Quantification of strain and charge co-mediated magnetoelectric coupling on ultra-thin Permalloy/PMN-PT interface, Sci. Rep. 4, 3688 (2014).

${ }^{59}$ Z. Zhou et al., Quantifying thickness-dependent charge mediated magnetoelectric coupling in magnetic/dielectric thin film heterostructures, Appl. Phys. Lett. 103, 232906 (2013).

${ }^{60} \mathrm{~T}$. Nozaki et al., Magnetization switching assisted by highfrequency-voltage-induced ferromagnetic resonance, Appl. Phys. Exp. 7, 073002 (2014).

${ }^{61}$ T. Nozaki et al., Electric-field-induced ferromagnetic resonance excitation in an ultrathin ferromagnetic metal layer, Nat. Phys. 8, 491 (2012).

${ }^{62} \mathrm{H}$. M. Zheng et al., Self-assembled growth of $\mathrm{BiFeO}_{3}-\mathrm{CoFe}_{2} \mathrm{O}_{4}$ nanostructures, Adv. Mater. 18, 2747 (2006).

${ }^{63} \mathrm{~W}$. Eerenstein et al., Comment on "epitaxial $\mathrm{BiFeO}_{3}$ multiferroic thin film heterostructures", Science 307, 1203a (2005).

${ }^{64}$ Y. H. Chu et al., Nanoscale domain control in multiferroic $\mathrm{BiFeO}_{3}$ thin films, Adv. Mater. 18, 2307 (2006).

${ }^{65} \mathrm{~T}$. Zhao et al., Electrical control of antiferromagnetic domains in multiferroic $\mathrm{BiFeO}_{3}$ films at room temperature, Nat. Mater. 5, 823 (2006).

${ }^{66}$ Y. H. Chu et al., Nanoscale control of domain architectures in $\mathrm{BiFeO}_{3}$ thin films, Nano Lett. 9, 1726 (2009).

${ }^{67} \mathrm{~S}$. H. Baek et al., Ferroelastic switching for nanoscale non-volatile magnetoelectric devices, Nat. Mater. 9, 309 (2010).

${ }^{68} \mathrm{~S}$. M. Wu et al., Reversible electric control of exchange bias in a multiferroic field-effect device, Nat. Mater. 9, 756 (2010).

${ }^{69}$ J. Wang, Epitaxial $\mathrm{BiFeO}_{3}$ multiferroic thin film heterostructures, Science 299, 1719 (2003).

${ }^{70}$ J. T. Heron et al., Deterministic switching of DzyaloshinskiiMoriya vector with electric field at room temperature, Nature 516, 370 (2014).

${ }^{71} \mathrm{~J}$. T. Heron et al., Electric-field-induced magnetization reversal in a ferromagnet-multiferroic heterostructure, Phys. Rev. Lett. 107, 217202 (2011).

${ }^{72}$ D. Qiu, K. Ashraf and S. Salahuddi, Nature of magnetic domains in an exchange coupled $\mathrm{BiFeO}_{3} / \mathrm{CoFe}$ heterostructure, Appl. Phys. Lett. 102, 112902 (2013).

${ }^{73}$ Z. Zhou et al., Probing electric field control of magnetism using ferromagnetic resonance, Nat. Commun. 6, 6082 (2015). 